\title{
Respostas cardiorrespiratória e metabólica do propofol nas formulações em emulsão lipídica ou microemulsão em gatas
}

\author{
Cardiorespiratory and metabolic answer with microemulsion and lipid emulsion \\ of propofol in cats
}

\author{
Renato Batista Tamanho ${ }^{\mathrm{I}}$ André Luís Corrêa ${ }^{\mathrm{I}}$ Aury Nunes de Moraes ${ }^{\mathrm{I}}$ Suzane Lilian Beier ${ }^{\mathrm{I}}$ \\ Doughlas Regalin ${ }^{\mathrm{I}}$ Felipe Hertzing Farias $^{\mathrm{I}}$ Nilson Oleskovicz ${ }^{\mathrm{I}}$
}

\section{RESUMO}

$O$ presente estudo objetiva avaliar os efeitos cardiorrespiratórios e metabólicos do propofol em emulsão lipídica e microemulsão em gatas. Foram utilizadas 12 gatas, hígidas, adultas, alocadas em dois grupos: microemulsão (MICRO, $n=6$ ) e emulsão lipídica (EMU, $n=6)$, os quais receberam propofol, na respectiva formulação, em dose suficiente para intubação. Em seguida, foram intubados, fornecendo-se oxigênio $100 \%$, em sistema sem reinalação de gases. Ato contínuo, iniciou-se a infusão de propofol na dose de $0,3 \mathrm{mg} \mathrm{kg}^{-1} \mathrm{~min}^{-1}$ durante 90 minutos. A dose necessária para indução foi de $9,5 \pm 1,3 \mathrm{mg} \mathrm{kg}^{-1}$ e $10 \pm 1 \mathrm{mg} \mathrm{kg}^{-1}$ para MICRO e EMU, respectivamente. Os valores de pressão arterial sistólica (PAS), pressão arterial média (PAM), pressão arterial diastólica (PAD) e pH foram menores, em todos os momentos, no EMU em relação ao MICRO; a f do EMU foi menor de T30 até $T 75$ em relação ao MICRO. A PaCO do EMU foi maior de T15 até T90. Os tempos de extubação, decúbito esternal, deambulação e recuperacão total foram de 40,6 $\pm 30,7 ; 91 \pm 37,5 ; 134,5 \pm 54,5$

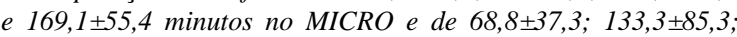
$171,3 \pm 77,1$ e 233,1 $\pm 60,6$ minutos no EMU, respectivamente. Houve aumento da enzima Alanina Aminotransferase de 12 às $72 \mathrm{~h}$ no EMU e de 48 às $72 \mathrm{~h}$ no MICRO. O propofol em microemulsão apresentou características clínicas de indução e manutenção, bem como efeitos metabólicos semelhantes à formulação em emulsão lipídica. A formulação em microemulsão proporcionou maior estabilidade cardiovascular e respiratória para indução e infusão contínua em gatas hígidas.

Palavras-chave: propofol, microemulsão, emulsão lipídica, gatas.

\section{ABSTRACT}

The aim of the present study was to evaluate the cardiorespiratory and metabolic effects from lipid emulsion and microemulsion of propofol in cats. Twelve healthy adult cats were included, and divided into two groups: microemulsion group (MICRO, $n=6)$ and lipid emulsion (EMU, $n=6)$, where they received propofol in the respective formulation, in a dose sufficient for intubation. The animals were then intubated and provided with $100 \%$ oxygen through a non-rebreathing circuit. Immediately after, the infusion of propofol was initiated $\left(0.3 \mathrm{mg} \mathrm{kg} \mathrm{kg}^{-1}\right.$ $\mathrm{min}^{-1}$ ) and maintained for 90 minutes. The dose required for induction was $9.5 \pm 1.3 \mathrm{mg} \mathrm{kg}^{-1}$ and $10 \pm 1 \mathrm{mg} \mathrm{kg}^{-1}$ in MICRO and $E M U$, respectively. The SAP, MAP, DAP and $p H$ values were lower in all moments in EMU when compared to MICRO; the RR in EMU was lower from T30 to T75 in comparison to MICRO. The $\mathrm{paCO}_{2}$ was greater in the EMU from T15 to T90. The times to extubation, sternal recumbency, ambulation and total recovery were $40.6 \pm 30.7,91 \pm 37.5,134.5 \pm 54.5$ and $169.1 \pm 55.4$ minutes in MICRO and $68.8 \pm 37.3,133.3 \pm 85.3,171.3 \pm 77.1$ and $233.1 \pm 60.6$ minutes in EMU, respectively. There was an increase of the enzyme alanine aminotransferase from 12 to 72 hours in EMU and in MICRO from 48 to 72 hours. The propofol in microemulsion presents clinical characteristics of induction and maintenance, and metabolic effects similar to the formulation in lipid emulsion. The microemulsion formulation provides a better cardiovascular and respiratory stability for induction and continuous infusion in healthy cats.

Key words: propofol, microemulsion, lipid emulsion, cats.

\section{INTRODUÇÃO}

O desenvolvimento de novos fármacos e/ ou novas formulações é responsável pelo surgimento de lacunas acerca dos possíveis efeitos sobre as variáveis fisiológicas e metabólicas nas diversas espécies. Nesse sentido, destacam-se os estudos com diferentes formulações de propofol em felinos, pois se sabe que essas formulações apresentam um padrão farmacocinético e farmacodinâmico diferenciado, conforme demonstram estudos de BESTER (2009) e CLEALE et al. (2009).

IDepartamento de Medicina Veterinária, Centro de Ciências Agroveterinárias (CAV), Universidade do Estado de Santa Catarina (UDESC), Av. Luiz de Camões, 2090, 88520-000, Lages, SC, Brasil. E-mail: a2no@ cav.udesc.br. *Autor para correspondência. 
O propofol (2,6-diisopropilfenol) é um derivado alquil-fenólico, de baixa solubilidade em água. A maioria das apresentações ainda é comercializada na concentração $1 \%$ e consiste em uma emulsão fina, contendo óleo de soja, fosfolipídios de ovo purificado e glicerol, conferindo à solução um aspecto leitoso com pH entre 7 e 8,5 (SANO et al., 2003). A presença de produtos orgânicos na formulação em emulsão lipídica e a ausência de antimicrobianos torna essa solução mais sujeita à contaminação. Algumas formulações mais recentes a base de nano partículas já estão disponíveis comercialmente.

O propofol em microemulsão apresentase como uma formulação óleo/água, sendo a fração oleosa constituída pelo propofol, pronta para uso e altamente estável, cujas partículas hidrofóbicas dispersas apresentam tamanho reduzido (1-50nm), com aspecto transparente e viscosidade comparável à de uma solução aquosa. Essa formulação apresenta um único surfactante do grupo constituído por estearatos de polietilenoglicol. Algumas das vantagens potenciais da utilização desse tipo de formulação diferenciada incluem uma maior vida de prateleira, devido à estabilidade termodinâmica, e uma reduzida propensão ao crescimento bacteriano, devido à ausência do óleo de soja como nutriente (MOREY et al., 2006).

O objetivo do presente estudo foi avaliar a utilização de uma nova formulação de propofol em microemulsão para indução e manutenção anestésica em gatos. Para tanto, avaliaram-se os efeitos cardiorrespiratórios, hemogasométricos, metabólicos e o índice biespectral, bem como os parâmetros clínicos e possíveis efeitos colaterais decorrentes da utilização do propofol em emulsão lipídica ou microemulsão em gatos domésticos.

\section{MATERIAL E MÉTODOS}

Foram utilizadas 12 gatas, comprovadamente hígidas, mestiças, com idade entre 1 e 4 anos e peso médio de $2,6 \pm 0,4 \mathrm{~kg}$, as quais foram submetidas a jejum alimentar de 12 horas e hídrico de seis. Foi realizada colheita de sangue pela veia jugular para aferição dos valores basais (0h) de hemograma e função e/ou lesão hepática (glicose, colesterol, albumina, Gama Glutamil Transferase (GGT), Fosfatase Alcalina (FA) e Alanina Aminotransferase (ALT); e função renal (ureia e creatinina); sendo esta repetida em $6,12,24,48$ e 72 horas após o término do procedimento experimental. Em ato contínuo, o sevoflurano foi administrado por meio de máscara facial conectado ao sistema sem reinalação de gases com o vaporizador ajustado a 5V\%, diluído em oxigênio a $100 \%$ com fluxo de $4 \mathrm{~L} \mathrm{~min}^{-1}$. Após a perda do reflexo laringotraqueal, os animais foram intubados e conectados ao sistema sem reinalação de gases e o vaporizador ajustado para 1,5 a 2,0CAM.

Foi realizada anestesia local com $0,5 \mathrm{~mL}$ de Lidocaína na região da artéria femoral, para posterior dissecação e introdução de um cateter Intracath 18G para mensuração da PAS, PAM e PAD, por meio de monitor multiparamétrico, bem como para colheita de sangue para análise hemogasométrica. Introduziuse também um cateter de mesmo calibre na veia jugular esquerda, para mensuração da pressão venosa central (PVC) pelo mesmo monitor. Ademais, foi introduzido um cateter $22 \mathrm{G}$ na veia cefálica direita e esquerda, para administração de fluidoterapia $(\mathrm{NaCl}$ $0,9 \%$ na taxa de $5 \mathrm{~mL} \mathrm{~kg}^{-1} \mathrm{~h}^{-1}$ ) e infusão dos fármacos utilizados, respectivamente. Após o término da anestesia inalatória, aguardaram-se 30 minutos para completa recuperação dos animais.

Nesse momento, os animais foram alocados, aleatoriamente, em dois grupos: microemulsão (MICRO, n=6), os quais receberam propofol em microemulsão (Propovet ${ }^{\circledR}\left(10 \mathrm{mg} \mathrm{mL}^{-1}\right)$, Ourofino Saúde Animal Ltda; Brasil); e emulsão (EMU, n=6), os quais receberam propofol em emulsão lipídica (Propovan ${ }^{\circledR}\left(10 \mathrm{mg} \mathrm{mL}^{-1}\right)$, Cristália; Brasil). O período de administração do fármaco foi de 30 segundos, ambos em dose suficiente para perda dos reflexos laringotraqueais e tônus mandibular, permitindo assim a intubação. A sonda foi conectada a um sistema sem re-inalação de gases, com fluxo de oxigênio a $100 \%$ de $4 \mathrm{~L} \mathrm{~min}^{-1}$. Após a indução, iniciouse a manutenção anestésica com a mesma formulação de propofol utilizada na indução, por meio de bomba de infusão de seringa, na taxa de $0,3 \mathrm{mg} \mathrm{kg}^{-1} \mathrm{~min}^{-1}$, durante 90 minutos.

Os parâmetros avaliados foram: frequência cardíaca (FC), através do traçado do eletrocardiograma no monitor multiparamétrico; frequência respiratória $(f)$, pela leitura no analisador de gases (sensor conectado entre a sonda traqueal e o circuito anestésico); PAS, PAM, PAD, PVC, por meio do monitor multiparamétrico, temperatura retal (TR) e índice biespectral (BIS). O posicionamento do sensor pediátrico do BIS foi adaptado da metodologia utilizada por GUERRERO \& NUNES (2003), em cães. Os valores de BIS eram registrados apenas quando os valores do Sinal Eletromiográfico (EMG) encontravam-se abaixo de 50 e os do Indicador de Qualidade de Sinal (SQI), acima de 50.

A mensuração dos parâmetros descritos acima foi realizada em T-10 (basal), aferida 30 
minutos após o término da anestesia inalatória; T0 (após indução anestésica e início da infusão contínua); e, em T15, T30, T45; T60; T75; e T90, correspondentes a 15, 30, 45, 60, 75 e 90 minutos após o início da infusão, respectivamente. As variáveis hemogasométricas (Pressão Parcial de Oxigênio no Sangue Arterial - $\mathrm{PaO}_{2}$. Pressão Parcial de Dióxido de Carbono no Sangue Arterial - $\mathrm{PaCO}_{2}$; Potencial de Hidrogênio - $\mathrm{pH}$; Bicarbonato - $\mathrm{HCO}_{3}$; Déficit de Base - DB; e Saturação de Oxihemoglobina no Sangue Arterial - $\mathrm{SaO}_{2}$ ) foram aferidas em: T-10, T0, T15, T30, T60 e T90. Avaliou-se também qualidade de indução, descrevendo-se todos os efeitos adversos observados, tempos de extubação (TE), para decúbito esternal (TDE), para deambulação (TDEA) e de recuperação total (TRT). Os dados entre tempos dentro de cada grupo foram submetidos à Análise de Variância de uma via com Repetições Múltiplas (ANOVA-RM), seguidas pelo Teste de Student Newman Keuls $(\mathrm{P} \leq 0,05)$. Entre grupos, utilizou-se o Teste $t$-Student $(\mathrm{P} \leq 0,05)$.

\section{RESULTADOS E DISCUSSÃO}

A dose para indução anestésica no MICRO foi de $9,5 \pm 1,3 \mathrm{mg} \mathrm{kg}^{-1}$ e no EMU de $10 \pm 1,0 \mathrm{mg} \mathrm{kg}^{-1}$. Todos os animais apresentaram indução rápida e suave, sem sinais de excitação ou qualquer outro efeito indesejável. Apesar das diferenças entre as formulações, as doses de indução foram semelhantes em ambos os grupos, da mesma forma como descrito por SANO et al. (2003) e CLEALE et al. (2009).

No EMU, houve redução da FC de aproximadamente $24 \%$, imediatamente após indução anestésica, perdurando até o final das avaliações (T90), apresentando redução significativa dos valores médios em relação ao basal. Por outro lado, no MICRO, a redução foi de aproximadamente $13 \%$, não sendo observada redução significativa em relação ao basal. Esses dados estão de acordo com os descritos por HASKINS (2006), o qual afirma que o propofol é um depressor da contratilidade cardíaca. Nesse sentido, pode-se especular que os excipientes utilizados na formulação em emulsão lipídica podem ter contribuído para maior depressão cardíaca observada no EMU. Os valores de PAS, PAM e PAD no EMU comportaram-se de maneira semelhante à FC, apresentando redução de aproximadamente $30 \%$, em relação ao basal, nos momentos T30 e T45, enquanto que os animais do MICRO apresentaram valores médios mais próximos aos fisiológicos, demonstrando assim uma maior estabilidade cardiovascular desse grupo (Tabela 1 e
Figura 1). Os valores médios de PAS, PAM e PAD do MICRO não apresentaram diferença significativa em relação ao basal e apresentaram valores médios significativamente maiores em relação ao EMU, durante todos os momentos após a indução.

A redução da pressão arterial decorrente da administração de propofol também foi descrita em cães por CORRÊA et al. (2010) e em gatos por CLEALE et al. (2009). DOURSOUT et al. (2002) atribuíram a queda da pressão arterial à estimulação do óxido nítrico, que pode estar mais relacionada ao veículo da emulsão lipídica, utilizada na formulação de propofol administrada em seu estudo (Intralipid ${ }^{\circledR}$ ), do que ao princípio ativo em si, podendo inferir que a utilização de formulações diferenciadas podem apresentar efeitos diferenciados. De maneira semelhante, CORRÊA et al. (2010), ao utilizarem, em cães, o mesmo fármaco em microemulsão do presente estudo, obtiveram resultados semelhantes para as mesmas variáveis, comprovando que, além dos veículos envolvidos, as respostas para um mesmo fármaco podem diferir entre espécies.

Não foram observadas diferenças significativas para a PVC entre os grupos, mantendose dentro dos limites fisiológicos para espécie. De certa forma, o referido episódio não era esperado, principalmente no EMU, visto que, nesse grupo, a redução dos valores de PAM e FC foi mais intensa. Soma-se ainda o fato de que o propofol promove redução significativa da resistência vascular sistêmica, através da inibição de mecanismos vasopressores medulares e redução da atividade dos barorreceptores periféricos (MUIR \& GADAWSKI, 2002).

A redução da $f$ foi mais evidente imediatamente após a indução e ao longo dos 90 minutos de infusão no EMU, com redução máxima de até 56\% (T30), quando comparado ao grupo MICRO e com redução máxima de 35\% (T90) em relação ao momento basal. De maneira semelhante, CLEALE et al. (2009) demonstraram redução de aproximadamente $50 \%$ da $f$ em gatas anestesiadas com propofol, porém essa redução foi evidenciada tanto com a emulsão comercial, quanto com a microemulsão. Apesar da depressão evidenciada em ambos os grupos, não foi observada apneia em nenhum animal do presente estudo.

Os valores médios de $\mathrm{PaCO}_{2}$ foram maiores que o basal, em todos os momentos, em ambos os grupos. Tal ocorrência pode ser atribuída aos valores basais abaixo do fisiológico para espécie. Segundo MUIR (2009), valores reduzidos de $\mathrm{PaCO}_{2}$ podem ser atribuídos ao estresse e ansiedade dos animais, ocasionando hiperventilação. Por outro 
Tabela 1- Valores médios e desvio padrão da frequência cardíaca ( $\mathrm{FC}$, bat $\left.\min ^{-1}\right)$, pressão arterial média (PAM, mmHg), pressão venosa central (PVC, $\mathrm{mmHg}$ ), frequência respiratória $\left(f, \mathrm{mov}_{\mathrm{min}^{-1}}\right)$, pressão arterial de dióxido de carbono $(\mathrm{PaCO}, \mathrm{mmHg}), \mathrm{pH}, \mathrm{déficit}$ de base (DB, mEq L $\left.{ }^{-1}\right)$, bicarbonato $\left(\mathrm{HCO}_{3}^{-}, \mathrm{mmol} \mathrm{L}^{-1}\right)$, índice bispectral (BIS), sinal eletromiográfico (EMG), qualidade do sinal (SQI), em gatas submetidas à indução e manutenção anestésica com propofol em microemulsão (M; n=6) ou em emulsão lipídica $(\mathrm{E} ; \mathrm{n}=6)$.

\begin{tabular}{|c|c|c|c|c|c|c|c|c|c|}
\hline & & $\mathrm{T}-10$ & T0 & $\mathrm{T} 15$ & $\mathrm{~T} 30$ & $\mathrm{~T} 45$ & T60 & $\mathrm{T} 75$ & T90 \\
\hline \multirow{2}{*}{$\mathrm{FC}$} & $\mathrm{M}$ & $208 \pm 25$ & $181 \pm 33$ & $185 \pm 23$ & $179 \pm 24$ & $189 \pm 29$ & $181 \pm 19$ & $183 \pm 14$ & $177 \pm 14$ \\
\hline & $\mathrm{E}$ & $221 \pm 22$ & $167 \mathrm{~A} \pm 25$ & $164 \mathrm{~A} \pm 23$ & $157 \mathrm{~A} \pm 21$ & $161 \mathrm{~A} \pm 24$ & $168 \mathrm{~A} \pm 26$ & $169 \mathrm{~A} \pm 18$ & $179 \mathrm{~A} \pm 8$ \\
\hline \multirow{2}{*}{ PAM } & $\mathrm{M}$ & $142 \pm 6$ & $145 \mathrm{a} \pm 14$ & $133 a \pm 28$ & $148 \mathrm{a} \pm 24,0$ & $150 \mathrm{a} \pm 18,7$ & $151 \mathrm{a} \pm 14,0$ & $146 \mathrm{a} \pm 13,7$ & $140 \mathrm{a} \pm 18,0$ \\
\hline & $\mathrm{E}$ & $126 \pm 19$ & $101 b \pm 29$ & $92 \mathrm{Ab} \pm 33$ & $89 \mathrm{Ab} \pm 29$ & $89 \mathrm{Ab} \pm 27$ & $93 \mathrm{Ab} \pm 24$ & $102 \mathrm{~A} \mathrm{~b} \pm 28$ & $101 \mathrm{Ab} \pm 27$ \\
\hline \multirow{2}{*}{ PVC } & $\mathrm{M}$ & $0,1 \pm 2,4$ & $-1,0 \pm 1,0$ & $-1,3 \pm 1,2$ & $-1,5 \pm 1,6$ & $-1,6 \pm 1,6$ & $-1,8 \pm 1,1$ & $-1,5 \pm 1,5$ & $-1,5 \pm 1,0$ \\
\hline & $\mathrm{E}$ & $0,4 \pm 0,8$ & $0,2 \pm 1,7$ & $-0,6 \pm 1,9$ & $-0,8 \pm 2,0$ & $-1,8 \pm 1,9$ & $-2,0 \mathrm{~A} \pm 1,7$ & $-1,8 \pm 2,0$ & $-2,2 \mathrm{~A} \pm 1,6$ \\
\hline \multirow{2}{*}{$f$} & $\mathrm{M}$ & $39 \pm 19$ & $33 \pm 8$ & $31 \pm 9$ & $30 \mathrm{a} \pm 8$ & $29 a \pm 6$ & $28 \mathrm{a} \pm 5$ & $27 a \pm 5$ & $25 \mathrm{~A} \pm 5$ \\
\hline & $\mathrm{E}$ & $37 \pm 6$ & $24 \mathrm{~A} \pm 9$ & $21 \mathrm{~A} \pm 9$ & $18 \mathrm{Ab} \pm 7$ & $16 \mathrm{Ab} \pm 7$ & $16 \mathrm{Ab} \pm 7$ & $16 \mathrm{Ab} \pm 6$ & $18 \mathrm{~A} \pm 7$ \\
\hline \multirow{2}{*}{$\mathrm{PaCO}_{2}$} & $\mathrm{M}$ & $26 \pm 2$ & $38 \mathrm{~A} \pm 5$ & $35 \mathrm{Aa} \pm 5$ & $33 \mathrm{Aa} \pm 5$ & - & $33 \mathrm{Aa} \pm 5$ & - & $35 \mathrm{Aa} \pm 5$ \\
\hline & $\mathrm{E}$ & $27 \pm 2$ & $41 \mathrm{~A} \pm 6$ & $45 \mathrm{Ab} \pm 8$ & $48 \mathrm{Ab} \pm 10$ & - & $50 \mathrm{Ab} \pm 11$ & - & $46 \mathrm{Ab} \pm 8$ \\
\hline \multirow{2}{*}{$\mathrm{pH}$} & M & $7,38 \pm 0,06$ & $7,28 \mathrm{~A} \pm 0,05$ & $7,33 \mathrm{Aa} \pm 0,04$ & $7,35 \mathrm{a} \pm 0,03$ & - & $7,34 a \pm 0,02$ & - & $7,33 \mathrm{Aa} \pm 0,01$ \\
\hline & $\mathrm{E}$ & $7,36 \pm 0,03$ & $7,25 \mathrm{~A} \pm 0,06$ & $7,24 \mathrm{Ab} \pm 0,06$ & $7,22 \mathrm{Ab} \pm 0,06$ & - & $7,23 \mathrm{Ab} \pm 0,07$ & - & $7,23 \mathrm{Ab} \pm 0,05$ \\
\hline \multirow{2}{*}{ DB } & $\mathrm{M}$ & $-8,9 \pm 1,6$ & $-8,6 \pm 1,7$ & $-6,3 \pm 1,3 \mathrm{~A}$ & $6,2 \pm 1,1 \mathrm{Aa}$ & - & $-6,3 \pm 1,3 \mathrm{~A}$ & - & $-6,4 \pm 1,4 \mathrm{Aa}$ \\
\hline & $\mathrm{E}$ & $-8,1 \pm 1,4$ & $-8,8 \pm 1,5$ & $-8,20 \pm 1,5$ & $-8,3 b \pm 1,6$ & - & $-7,34 \pm 1,2$ & - & $-8,35 b \pm 1,7$ \\
\hline \multirow{2}{*}{$\mathrm{HCO}_{3}^{-}$} & $\mathrm{M}$ & $13,8 \pm 2,2$ & $17,3 \pm 1,8$ & $18,6 \mathrm{~A} \pm 1,7$ & $18,1 \mathrm{~A} \pm 1,7$ & - & $18,2 \mathrm{~A} \pm 2,2$ & - & $18,4 \mathrm{~A} \pm 2,1$ \\
\hline & $\mathrm{E}$ & $15,4 \pm 1,0$ & $17,8 \mathrm{~A} \pm 1,4$ & $18,9 \mathrm{~A} \pm 1,8$ & $19,2 \mathrm{~A} \pm 2,5$ & - & $20,4 \mathrm{~A} \pm 2,1$ & - & $19,0 \mathrm{~A} \pm 1,5$ \\
\hline \multirow[t]{2}{*}{ BIS } & $\mathrm{M}$ & - & $83 \pm 10$ & $77 \pm 6$ & $80 \pm 9$ & $82 \pm 10$ & $82 \pm 7$ & $81 \pm 8$ & $82 \pm 11$ \\
\hline & $\mathrm{E}$ & - & $80 \pm 7$ & $79 \pm 10$ & $78 \pm 11$ & $78 \pm 14$ & $75 \pm 20$ & $74 \pm 13$ & $76 \pm 17$ \\
\hline \multirow[t]{2}{*}{ EMG } & M & - & $40 \pm 5$ & $36 \pm 3$ & $36 \pm 5$ & $39 \pm 5$ & $39 \pm 5$ & $38 \pm 6$ & $40 \pm 5$ \\
\hline & $\mathrm{E}$ & - & $37 \pm 3$ & $38 \pm 4$ & $37 \pm 4$ & $40 \pm 2$ & $37 \pm 3$ & $35 \pm 4$ & $35 \pm 3$ \\
\hline \multirow[t]{2}{*}{ SQI } & M & - & $79 \pm 13$ & $93 \pm 5$ & $87 \pm 18$ & $84 \pm 10$ & $82 \pm 12$ & $87 \pm 15$ & $78 \pm 18$ \\
\hline & $\mathrm{E}$ & - & $75 \pm 8$ & $87 \pm 5$ & $81 \pm 14$ & $79 \pm 12$ & $77 \pm 8$ & $77 \pm 7$ & $68 \pm 12$ \\
\hline
\end{tabular}

Letra A na linha indica valor diferente de T-10, Teste de Student Newman Keuls $(\mathrm{P} \leq 0,05)$. Letra minúscula diferente entre as linhas de uma mesma coluna indica valor diferente entre grupos, Teste $\mathrm{t}(\mathrm{P} \leq 0,05)$.

lado, pode-se observar que apenas o EMU apresentou valores acima do limite fisiológico para a espécie. Esse episódio pode ser justificado pelos menores valores de $f$ ao longo dos momentos no EMU, levando à redução da fração expirada de $\mathrm{CO}_{2}$ e ao seu acúmulo no organismo. Entre grupos, a $\mathrm{PaCO}_{2}$ do EMU foi maior de T15 até T90 em relação ao MICRO. A diferença apresentada pode ser atribuída à formulação do propofol em microemulsão, frente ao metabolismo diferenciado dos gatos, uma vez que esse episódio não ocorreu em estudo de CORRÊA et al. (2010), utilizando a mesma formulação em cães, bem como em outros estudos, utilizando diferentes formulações de propofol em cães (MOREY et al., 2006) e gatos (CLEALE et al., 2009).

No EMU, houve redução do pH em todos os momentos em relação ao basal, enquanto que, no MICRO, o $\mathrm{pH}$ foi menor apenas em T0, T15 e T90. Entre grupos, os valores de $\mathrm{pH}$ do EMU foram menores, quando comparados ao MICRO desde T15 até T90. Apesar de relevante, a análise isolada do $\mathrm{pH}$ não é bom indicativo para verificar se houve ou não alteração do equilíbrio ácido base, uma vez que esse parâmetro pode não estar alterado em caso de 


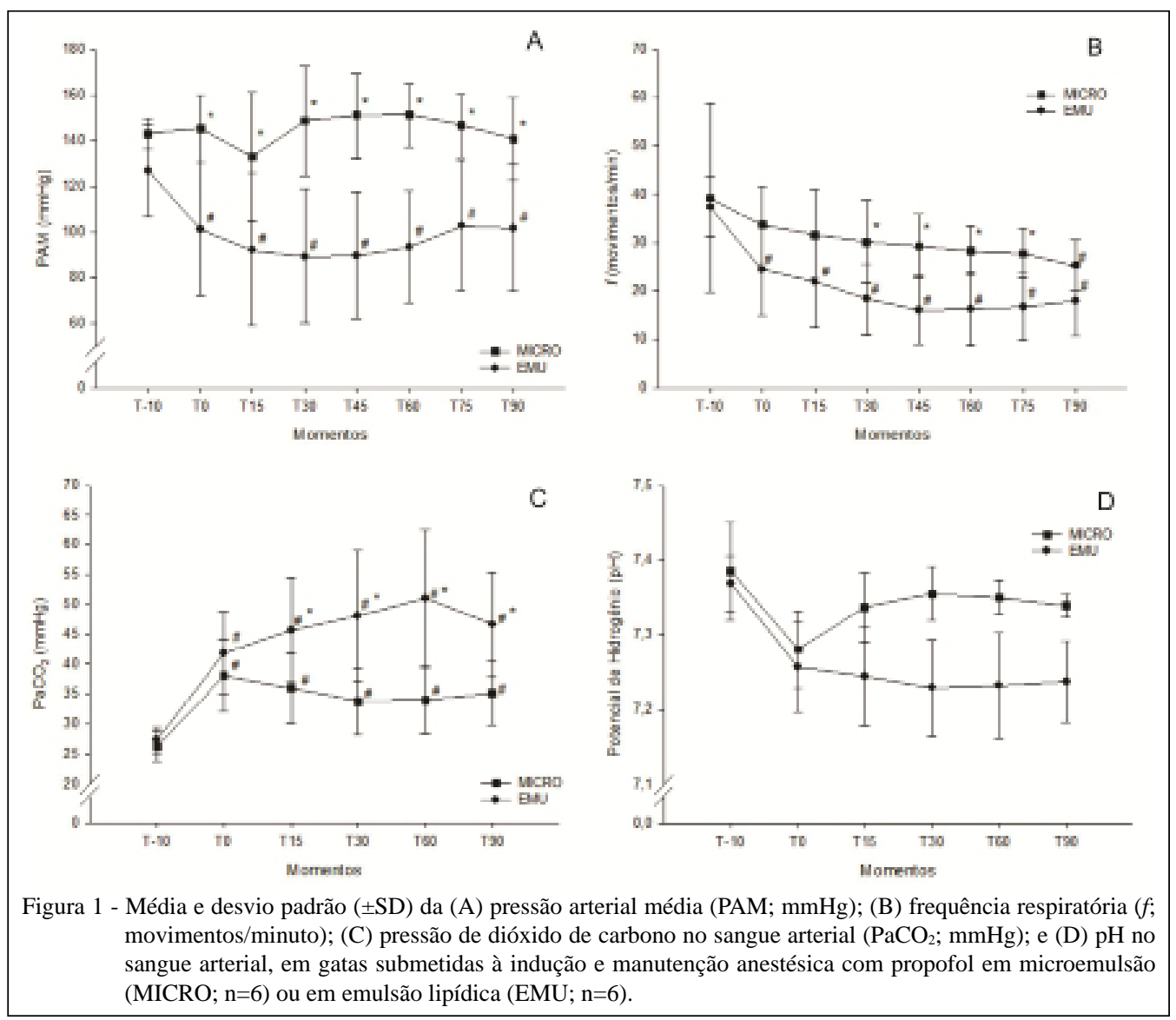

resposta compensatória pulmonar, renal, ou ainda em casos de distúrbios mistos. Diante desse cenário, fica evidente que uma das causas da redução do $\mathrm{pH}$ no EMU foi a depressão respiratória com redução da $f$ e aumento dos níveis de $\mathrm{CO}_{2}$ no sangue arterial, uma vez que, a partir de T0, a elevação dos níveis de $\mathrm{CO}_{2}$ se comportaram de maneira oposta ao $\mathrm{pH}$ sanguíneo, caracterizando um quadro de acidose respiratória.

Os valores de $\mathrm{HCO}_{3}$ - foram maiores em todos os momentos no EMU e em T15, T30, T60 e T90 no MICRO, em relação ao basal. Esse aumento pode ser atribuído à elevação da $\mathrm{PaCO}_{2}$ (SANO et al., 2003). Segundo JOHNSON \& MORAIS (2007), à medida que os tecidos produzem dióxido de carbono, ocorre equilíbrio imediato entre os compartimentos intracelulares e extracelulares. Dessa forma, o $\mathrm{CO}_{2} \mathrm{se}$ difunde rapidamente dos tecidos para os eritrócitos,

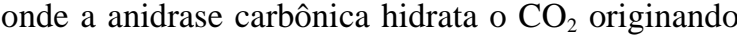
ácido carbônico.

Referente aos valores de DB, os animais do MICRO apresentaram menor DB desde T15 até T90, quando comparados a T-10. Entre grupos, o EMU apresentou maior DB em T30 e T90 em relação ao MICRO. Esse aumento acentuado indica consumo das reservas de $\mathrm{HCO}_{3}^{-}$e hemoglobina no sistema tampão, buscando normalizar o quadro de acidose respiratória existente nesse grupo, caracterizada pelo aumento da $\mathrm{PaCO}_{2}$ e presença de $\mathrm{pH}$ entre 6,8 e 7,2.

Os valores do BIS não apresentaram diferença significativa entre grupos ou entre os tempos, sendo que, no MICRO, os animais apresentaram valor médio do BIS de 80,8 $\pm 1,9$ e, no EMU, os valores médios foram de 77,1 $\pm 2,1$. Segundo GUERRERO \& NUNES (2003), os valores obtidos em ambos os grupos foram compatíveis com um quadro de sedação leve a profunda (70-90). Em contrapartida, as características clínicas dos animais eram condizentes com um plano anestésico mais profundo. Esses valores elevados podem ser atribuídos a uma atividade eletromiográfica aumentada, com valores médios mantidos próximos a 40, ao longo das observações, o que vai de encontro aos resultados obtidos por BEIER et al. (2009) e CORRÊA et al. (2010), os quais observaram uma forte correlação entre o EMG e o BIS durante a infusão de propofol em cães.

Os tempos de extubação, decúbito esternal, deambulação e de recuperação total foram de $40,6 \pm 30,7 ; 91 \pm 37,5 ; 134,5 \pm 54,5$ e $169,1 \pm 55,4$ minutos no MICRO e de $68,8 \pm 37,3 ; 133,3 \pm 85,3 ; 171,3 \pm 77,1$ e $233,1 \pm 60,6$ minutos no EMU, respectivamente. 
Embora não tenha ocorrido diferença estatística entre os parâmetros relacionados à recuperação dos animais, clinicamente, os animais do grupo MICRO apresentaram menores valores para todas essas variáveis. O elevado tempo de recuperação após longos períodos e/ou altas taxas de infusão podem ser reflexo de um efeito dose dependente, devido ao acúmulo do fármaco nos tecidos periféricos menos vascularizados (ESTIVALET et al., 2002), ou, ainda, pelo maior volume de distribuição e a menor taxa de eliminação do propofol, que são as principais diferenças farmacocinéticas observadas entre a espécie felina e as demais (BESTER, 2009).
Observou-se aumento, acima do limite fisiológico, de ALT de 12 até $72 \mathrm{~h}$ em ambos os grupos (Tabela 2), demonstrando um forte indicativo de que houve lesão hepática. KANEKO et al. (2008) afirmam que a atividade sérica de ALT aumenta há aproximadamente 12 horas após a lesão hepática e atinge o valor máximo cerca de um a dois dias após uma lesão aguda. A redução significativa nos valores de albumina ocorrida em ambos os grupos, em relação ao basal, não reflete um quadro de insuficiência hepática, pois, segundo KANEKO et al. (2008), tais valores mantiveram-se dentro da normalidade para a espécie. Corroborando tal achado, CENTER (2007)

Tabela 2- Valores médios e desvio padrão da contagem de alanino aminotransferase (ALT, $\mathrm{U} \mathrm{L}^{-1}$ ), gama glutamil transferase (GGT, $\left.\mathrm{U} \mathrm{L}^{-1}\right)$, fosfatase alcalina (FA, $\left.\mathrm{U} \mathrm{L}^{-1}\right)$, albumina (ALB, $\left.\mathrm{g} \mathrm{dL}^{-1}\right)$, colesterol $\left(\mathrm{COL}, \mathrm{mg} \mathrm{dL}^{-1}\right)$, glicose $\left(\mathrm{GLI}, \mathrm{mg} \mathrm{dL}^{-1}\right)$, ureia $\left(\mathrm{U}, \mathrm{mg} \mathrm{dL}^{-1}\right)$, creatinina (CRE, $\left.\mathrm{mg} \mathrm{dL}^{-1}\right)$, eritrócitos $\left(\mathrm{ERI}, \mathrm{x} 10^{6} \mu \mathrm{L}^{-1}\right)$, volume globular $(\mathrm{VG}, \%)$, Proteínas $\left(\mathrm{PT}\right.$, $\left.\mathrm{g} \mathrm{dl}{ }^{-1}\right)$, em gatas submetidas à indução e manutenção anestésica com propofol em microemulsão (M; n=6) ou em emulsão lipídica (E; n=6).

\begin{tabular}{|c|c|c|c|c|c|c|c|}
\hline & & $\mathrm{Oh}$ & $6 \mathrm{~h}$ & $12 \mathrm{~h}$ & $24 \mathrm{~h}$ & $48 \mathrm{~h}$ & $72 \mathrm{~h}$ \\
\hline \multirow{2}{*}{ ALT } & $\mathrm{M}$ & $51 \pm 25$ & $83 \mathrm{~A} \pm 29$ & $93 \mathrm{~A} \pm 30$ & $98 \mathrm{~A} \pm 27$ & $157 \mathrm{~A} \pm 47$ & $150 \mathrm{~A} \pm 37$ \\
\hline & $\mathrm{E}$ & $68 \pm 29$ & $78 \pm 20$ & $104 \pm 39$ & $110 \pm 32$ & $109 \pm 23$ & $112 \pm 53$ \\
\hline \multirow{2}{*}{ GGT } & $\mathrm{M}$ & $2,1 \pm 1,7$ & $2,3 \pm 2,6$ & $2,4 \pm 1,8$ & $2,6 \pm 1,1$ & $1,9 \pm 0,4$ & $3,3 \pm 1,3$ \\
\hline & $\mathrm{E}$ & $2,4 \pm 0,9$ & $3,5 \pm 1,7$ & $2,6 \pm 1,9$ & $2,6 \pm 1,8$ & $1,8 \pm 0,6$ & $2,8 \pm 2,0$ \\
\hline \multirow{2}{*}{ FA } & $\mathrm{M}$ & $50 \pm 14$ & $39 \pm 13$ & $40 \pm 10$ & $36 \pm 9$ & $29 \pm 7$ & $28 \pm 6$ \\
\hline & $\mathrm{E}$ & $47 \pm 26$ & $30 \pm 9$ & $37 \pm 12$ & $29 \pm 11,7$ & $35 \pm 16$ & $33 \pm 13$ \\
\hline \multirow{2}{*}{ ALB } & M & $3,3 \pm 0,2$ & $2,8 \mathrm{~A} \pm 03$ & $2,7 \mathrm{~A} \pm 0,2$ & $2,7 \mathrm{~A} \pm 0,2$ & $2,9 \mathrm{~A} \pm 0,1$ & $2,9 \mathrm{~A} \pm 0,1$ \\
\hline & $\mathrm{E}$ & $3,2 \pm 0,3$ & $2,8 \mathrm{~A} \pm 0,2$ & $2,8 \mathrm{~A} \pm 0,3$ & $2,8 \mathrm{~A} \pm 0,2$ & $2,6 \mathrm{~A} \pm 0,3$ & $2,6 \mathrm{~A} \pm 0,3$ \\
\hline \multirow{2}{*}{$\mathrm{COL}$} & $\mathrm{M}$ & $98 \pm 22$ & $61 \mathrm{~A} \pm 4$ & $68 \mathrm{~A} \pm 4$ & $89 \pm 10$ & $142 \mathrm{Aa} \pm 14$ & 149 Aa \pm 19 \\
\hline & $\mathrm{E}$ & $93 \pm 19$ & $72 \mathrm{~A} \pm 10$ & $76 \pm 14$ & $89 \pm 18$ & $108 \mathrm{Ab} \pm 20$ & $120 \mathrm{Ab} \pm 19$ \\
\hline \multirow{2}{*}{ GLI } & $\mathrm{M}$ & $102 \pm 21$ & $168 \mathrm{~A} \pm 12$ & $157 \mathrm{~A} \pm 21$ & $162 \mathrm{~A} \pm 23$ & $145 \mathrm{~A} \pm 29$ & $121 \mathrm{~A} \pm 25$ \\
\hline & $\mathrm{E}$ & $101 \pm 38$ & $182 \mathrm{~A} \pm 34$ & $179 \mathrm{~A} \pm 36$ & $168 \mathrm{~A} \pm 45$ & $164 \mathrm{~A} \pm 42$ & $153 \mathrm{~A} \pm 38$ \\
\hline \multirow{2}{*}{$\mathrm{U}$} & $\mathrm{M}$ & $55 \pm 8$ & $45 \mathrm{~A} \pm 3$ & $46 \mathrm{~A} \pm 7$ & $45 \mathrm{~A} \pm 6$ & $42 \mathrm{~A} \pm 2$ & $49 \pm 5$ \\
\hline & $\mathrm{E}$ & $61 \pm 7$ & $53 \pm 8$ & $65 \pm 21$ & $62 \pm 20$ & $67 \pm 41$ & $72 \pm 29$ \\
\hline \multirow{2}{*}{ CRE } & $\mathrm{M}$ & $1,2 \pm 0,1$ & $1,0 \mathrm{Aa} \pm 0,1$ & $0,8 \mathrm{~A} \pm 0,2$ & $0,8 \mathrm{~A} \pm 0,1$ & $1,0 \mathrm{~A} \pm 0,1$ & $0,9 \mathrm{~A} \pm 0,2$ \\
\hline & $\mathrm{E}$ & $1,4 \pm 0,3$ & $1,2 \mathrm{~b} \pm 0,2$ & $1,3 \pm 0,5$ & $1,3 \pm 0,6$ & $1,3 \pm 0,9$ & $1,2 \pm 0,4$ \\
\hline \multirow{2}{*}{ ERI } & $\mathrm{M}$ & $7,5 \pm 1,4$ & $6,8 \pm 0,7$ & $6,2 \pm 0,5$ & $6,7 \pm 1,4$ & $7,0 \pm 0,6$ & $6, \pm 0,7$ \\
\hline & $\mathrm{E}$ & $7,4 \pm 1,4$ & $6,7 \pm 0,3$ & $6,4 \pm 0,6$ & $6,2 \pm 0,2$ & $6,5 \pm 0,4$ & $5,8 \pm 1,3$ \\
\hline \multirow{2}{*}{ VG } & $\mathrm{M}$ & $38,3 \pm 5,3$ & $32,2 \mathrm{~A} \pm 3,8$ & $30,7 \mathrm{~A} \pm 3,4$ & $31,0 \mathrm{~A} \pm 5,5$ & $31,5 \mathrm{~A} \pm 3,9$ & $38,5 \mathrm{~A} \pm 3,6$ \\
\hline & $\mathrm{E}$ & $34,6 \pm 5,6$ & $32,7 \pm 1,5$ & $32,2 \pm 3,8$ & $32,2 \pm 3,3$ & $30,4 \pm 3,1$ & $27,1 \mathrm{~A} \pm 6,5$ \\
\hline \multirow{2}{*}{ PT } & M & $7,4 \pm 0,6$ & $6,1 \pm 0,2$ & $6,4 \pm 0,12$ & $6,4 \pm 0,3$ & $7,0 \pm 0,3$ & $6,8 \pm 0,8$ \\
\hline & $\mathrm{E}$ & $6,3 \pm 0,6$ & $6,3 \pm 0,3$ & $6,4 \pm 0,4$ & $6,4 \pm 0,5$ & $6,7 \pm 0,5$ & $6,5 \pm 1$ \\
\hline
\end{tabular}

Letra A na linha indica valor diferente de $0 \mathrm{~h}$, Teste de Student Newman Keuls $(\mathrm{P} \leq 0,05)$. Letra minúscula diferente entre as linhas de uma mesma coluna indica valor diferente entre grupos, Teste $t(P \leq 0,05)$. 
relata que os níveis de albumina podem reduzir em até $50 \%$, dentro de 24 horas, após períodos de jejum. Ademais, os valores de FA e GGT não apresentaram diferença significativa e mantiveram-se dentro dos valores preconizados para gatos, segundo KANEKO et al. (2008).

Observou-se redução nos valores de colesterol em seis e 12 horas no MICRO e em seis horas no EMU e aumento significativo em 48 e 72 horas em ambos os grupos, em relação ao basal. Entre grupos, os valores de colesterol foram menores no EMU, quando comparados ao MICRO em 48 e 72 horas após o término da infusão. Os valores de glicose foram maiores, em todos os momentos de ambos os grupos, quando comparados ao basal. Os valores médios de glicose e colesterol não apresentaram homogeneidade ao longo das avaliações, pois, baseando-se nos valores de referência de KANEKO et al. (2008), ficaram abaixo do limite, em ambos os grupos até $24 \mathrm{~h}$, e acima do intervalo para a espécie após 48h no MICRO. Essa ausência de homogeneidade dos valores pode ser atribuída à dieta alimentar e ao regime de fornecimento desta (MEYER et al., 1995).

Não foram observadas alterações relevantes nos parâmetros hematológicos e função renal entre os tempos ou entre os grupos avaliados. Entretanto, os valores médios de leucócitos e neutrófilos apresentaram-se acima do limite fisiológico para gatos, ao longo dos tempos em ambos os grupos. Essas alterações observadas podem ser decorrentes da ansiedade e esforço físico, em virtude da contenção dos animais, resultando na liberação de epinefrina, com consequente aumento do fluxo sanguíneo da microcirculação, levando à migração de leucócitos do compartimento marginal para o compartimento circulante (MEYER et al., 1995).

As diferenças encontradas nos parâmetros cardiovasculares do presente estudo podem estar relacionadas à diferença dos veículos das formulações utilizadas. Diante disso, fica evidenciada a necessidade da realização de estudos utilizando apenas os veículos das formulações testadas, em diferentes espécies, para que possamos afirmar quais excipientes estão envolvidos nas alterações cardiovasculares apresentadas.

\section{CONCLUSÃO}

O propofol em microemulsão apresentou efeitos clínicos e metabólicos semelhantes à formulação em emulsão lipídica comercialmente disponível, porém com maior estabilidade cardiovascular e respiratória para indução e manutenção anestésica em gatos.

\section{COMITÊ DE ÉTICA E BIOSSEGURANÇA}

Este estudo foi aprovado pelo Comitê de Ética e de Bem Estar Animal - CETEA - da instituição de origem (1.39/09).

\section{AGRADECIMENTOS}

À Universidade do Estado de Santa Catarina (UDESC), pelo auxílio do PROMOP. À Coordenação de Aperfeiçoamento de Pessoal de Nível Superior (CAPES), pela concessão da bolsa.

\section{REFERÊNCIAS}

BEIER, S.L. et al. Effect of remifentanil on requirements for propofol administered by use of a target-controlled infusion system for maintaining anesthesia in dogs. American Journal of Veterinary Research, v.70, n.6, p.703-709, 2009. Disponível em: <http://www.ncbi.nlm.nih.gov/pubmed/19496658>. Acesso em: 23 fev. 2012. doi: 10.2460/ajvr.70.6.703.

BESTER, L. Pharmacokinetics of propofol in cats. 2009 72f. Tese (Doctor in Department of Companion Animal Clinical Studies) - Faculty of Veterinary Science University of Pretoria Pretoria, South Africa. Disponível em: <http://upetd.up.ac.za/ thesis/available/etd-03032010-184513/unrestricted/dissertation. pdf>. Acesso em: 23 fev. 2012.

CENTER, S.A. Distúrbios hidroeletrolíticos e ácido-básicos na doença hepática. In: DIBARTOLA, S.P. Anormalidade de fluidos, eletrólitos e equilíbrio ácido-básico na clínica de pequenos animais. 3.ed. São Paulo: Roca, 2007. Cap.19, p.421-460.

CLEALE, M.R. et al. Pharmacokinetic and pharmacodynamic evaluation of Propofol administered to cats in a novel, aqueous, nano-droplet formulation or as an oil-in-water macroemulsion. Journal Veterinary Pharmacology Theraphy, n.32, p.436445, 2009. Disponível em: <http://onlinelibrary.wiley.com/ doi/10.1111/j.1365-2885.2009.01059.x/full>. Acesso em: 23 fev. 2012. doi: $10.1111 / \mathrm{j} .1365-2885.2009 .01059$.x.

CORRÊA, A.L. Avaliação hemodinâmica e metabólica de uma nanoemulsão de propofol em cães. 2010. 150f. Dissertação (Mestrado Ciência Animal) - Centro de Ciências Agroveterinárias, Universidade do Estado de Santa Catarina, Lages, SC. Disponível em: <http://cienciaanimal.cav.udesc.br/dissertacoes/Andre_Correa. pdf>. Acesso em: 23 fev. 2012.

DOURSOUT, M.F. et al. Role of propofol and its solvent, Intralipid, in nitric oxide-induced peripheral vasodilatation in dogs. British Journal of Anaesthesia, v.89, n.3, p.492-498, 2002. Disponível em: <http://www.ncbi.nlm.nih.gov/pubmed/12402731>. Acesso em: 23 fev. 2012.

ESTIVALET, F.F. et al. Remifentanil associado ao propofol ou sevofluorano para a colecistectomia videolaparoscópica: estudo comparativo. Revista Brasileira de Anestesiologia, v.52, p.385393, 2002. Disponível em: <http://www.scielo.br/pdf/rba/v52n4/ v52n4a01.pdf>. Acesso em: 23 fev. 2012. doi: 10.1590/S003470942002000400001

GUERRERO, P.N.H.; NUNES, N. Monitoramento do índice biespectral em cães. Semina: Ciências Agrárias, v.24, n.1, p.163170, 2003. Disponível em: <http://www.uel.br/revistas/uel/index. php/semagrarias/article/view/2144/1839>. Acesso em: 23 fev. 2012. HASKINS, S.C. Comparative cardiovascular and pulmonary 
effects of sedatives and anesthetic agents and anesthetic drug selection for the trauma patient. Journal of Veterinary Emergency and Critical Care, v.16, n.4, p.300-328, 2006. Disponível em: <http://onlinelibrary.wiley.com/doi/10.1111/ j.1476-4431.2006.00188.x/full>. Acesso em: 23 fev. 2012. doi: 10.1111/j.1476-4431.2006.00188.x.

JOHNSON, R.A.; de MORAIS, H.A. Distúrbios ácido-básicos respiratórios. In: DIBARTOLA, S.P. Anormalidade de fluidos, eletrólitos e equilíbrio ácido-básico na clínica de pequenos animais. 3.ed. São Paulo: Roca, 2007. Cap.11, p.270-282.

KANEKO, J.J. et al. Clinical biochemistry of domestic animals. 6.ed. London: Academic, 2008. 928p.

MEYER, D.J. et al. Medicina de laboratório veterinária Interpretação e diagnóstico. São Paulo: Roca, 1995. p 320.

MOREY, T. E. et al. Anesthetic properties of a propofol microemulsion in dogs. Anesthesia and Analgesia, v.103, p.882-887, 2006. Disponível em: <http://www.ncbi.nlm.nih.gov/ pubmed/17000798>. Acesso em: 23 fev. 2012. doi: 10.1213/01. ane.0000237126.57445.80.

MUIR III, W.W. Dor e estresse. In: GAYNOR, J.S.; MUIR III, W.W. Manual de controle da dor em medicina veterinária. 2.ed. São Paulo: Med Vet, 2009. Cap.3, p.42-56.

MUIR III, W.W.; GADAWSKI, J.E. Cardiovascular effects of a high dose of romifidine in propofol-anesthetized cats. American Journal of Veterinary Research, v.63, n.9, p.12411246, 2002. Disponível em: <http://www.ncbi.nlm.nih.gov/ pubmed/12224853>. Acesso em: 10 ago. 2012.

SANO, T. et al. Clinical usefulness of propofol as an anesthetic induction agent in $\operatorname{dogs}$ and cats. Journal of Veterinary Medicine Science, v.65, n.5, p.641-643, 2003. Disponível em: <http://www.jstage.jst.go.jp/article/jvms/65/5/641/_pdf>. Acesso em: 23 fev. 2012. 